\title{
ARTICLE
}

\section{Heterogeneity effect on neutron shielding in borated concrete and Monte Carlo-based cross section homogenization method for particle dispersed media}

\author{
Toshihiro Yamamoto* \\ Kyoto University, 2-1010 Asashironishi, Kumatori-cho, Sennan-gun, Osaka-fu, 590-0494
}

\begin{abstract}
A Monte Carlo-based radiation transport calculation method to treat media containing randomly and uniformly dispersed spherical particles was developed by Shmakov et al. The method (hereinafter referred to as "SLD method") formulates effective homogenized cross sections for particle dispersed media. This paper discusses the applicability of the SLD method to neutron shielding in an ordinary concrete and a low-activation concrete containing small $\mathrm{B}_{4} \mathrm{C}$ particles. If the absorber particles are not small enough, the heterogeneity effect needs to be treated for accurate dose or activation estimations. The direct heterogeneous representation of the small particles requires long computing time. The use of the SLD method could considerably reduce the computational burdens without reducing its accuracy. The heterogeneity effect on neutron shielding is significant below $10 \mathrm{eV}$. The homogeneous calculation underestimates the neutron absorption rate of ${ }^{23} \mathrm{Na}$ by $11 \%$ in the ordinary concrete and of ${ }^{151} \mathrm{Eu}$ by $14 \%$ in the low-activation concrete containing $14-\mu \mathrm{m}$-diameter $\mathrm{B}_{4} \mathrm{C}$ particles. MCNP $4 \mathrm{C}$ calculations with the SLD method agree very well with the direct heterogeneous calculations. The SLD method improves the figure of merit by a factor of 100 with respect to the direct heterogeneous calculation.
\end{abstract}

Keywords: Monte Carlo; shielding; neutron; concrete; absorber; heterogeneity

\section{Introduction}

A Monte Carlo-based radiation transport calculation method to treat media containing randomly and uniformly dispersed spherical particles such as fuels for high-temperature gas-cooled reactors (HTGR) was developed by Shmakov, Lyutov and Dean [1]. The method formulates effective homogenized microscopic and macroscopic cross sections for particle dispersed media (hereinafter referred to as "SLD method"). The SLD method can easily be incorporated into continuous-energy Monte Carlo codes with slight modifications. Unlike direct representation of a heterogeneous medium containing dispersed particles, this method can perform radiation transport calculations without a time-consuming trajectory tracking for a large number of dispersed particles, and the computational burdens are much reduced.

Yamamoto et al. corrected some drawbacks in the original SLD method and enhanced its versatility [2-4]. The modified SLD method was applied to some criticality calculations with MCNP 4C [5] in which fuel or absorber particles are randomly and uniformly distributed in the matrix materials, e.g., $\mathrm{Pu}$ spots in a MOX fuel pellet.

All applications of the SLD method to date have

*Corresponding author. Email: toshihiro.yamamoto223@gmail.com targeted criticality calculations. The SLD method has not been validated yet for fixed source calculations. In a deep penetration calculation, for example, a small calculation error caused by the SLD method in an upstream part of the shielding material would be enlarged in the downstream part, which may yield unacceptable erroneous doses. This paper discusses the applicability of the SLD method to neutron shielding calculations.

A neutron shielding with the use of neutron absorber is one of examples where the heterogeneity effect of small dispersed particles needs to be considered. Usually, it is assumed that neutron absorber can be homogeneously mixed with the matrix of the shielding material. If the absorber particles are not small enough, however, the heterogeneity effect needs to be treated for accurate dose or activation estimations. While the Monte Carlo calculation can be performed by explicitly distributing a large number of particles in the shielding material, the direct representation of the particles usually requires long computing time. The use of the SLD method could considerably reduce the computational burdens without reducing its accuracy.

As the radiation shielding materials used for nuclear facilities, a variety of borated concretes have been developed to reduce the induced radioactivity within the concrete [6]. In accelerator facilities, to reduce the activity of ${ }^{24} \mathrm{Na}$ induced by neutron absorption of ${ }^{23} \mathrm{Na}$ is 
important in term of the dose control. In the biological shielding concretes used for power reactors, the activation of impurities such as Co and Eu would be a key issue in terms of its clearance level. This paper applies the modified SLD method to neutron shielding calculations in the borated concretes used in nuclear facilities. The heterogeneity effect of the $\mathrm{B}_{4} \mathrm{C}$ particles on the induced activities is discussed.

\section{Theory of the SLD method}

The theory of the SLD method is briefly described here. For more details, see the references [1-4]. Suppose that spherical particles with the diameter of $D$ are dispersed uniformly in a slab with the thickness of $L$ $(>D)$. The effective homogenized macroscopic total cross section of the particle-dispersed slab is given by [1-4]

$$
\bar{\Sigma}=\Sigma_{m}-\frac{1}{L} \ln [1-q+q J(-\Delta \Sigma, D)],
$$

where

$$
J(x, y)=\frac{2}{y^{2}}\left(\frac{1}{x^{2}}+\left(\frac{y}{x}-\frac{1}{x^{2}}\right) \exp (x y)\right),
$$

$\Sigma_{m}=$ total cross section of the matrix, $q=1.5 \alpha, \alpha=$ volume fraction of the particle, $\Delta \Sigma=\Sigma_{a}-\Sigma_{m}, \Sigma_{a}=$ total cross section of the particle. The effective microscopic cross section of a nuclide within the particle, $\sigma_{p}$, can be calculated by $\sigma_{p}=F_{p} \cdot \sigma$ where $\sigma=$ a microscopic cross section of any kind within the particle. The factor $F_{p}$, which represents the heterogeneity effect on the cross section of the particle, is given by

$$
F_{p}=\frac{\bar{\Sigma} P_{a}}{\alpha \Sigma_{a} P_{T}}
$$

where $P_{a}=$ probability that a neutron collides with the particle, $P_{T}=$ probability that a neutron collides with the medium with the thickness of $L$ (including the collision with the matrix and particle materials). In the same way, the effective microscopic cross section of a nuclide in the matrix, $\sigma_{m}$, can be calculated by $\sigma_{m}=F_{m} \cdot \sigma$ where

$$
F_{m}=\frac{\bar{\Sigma}\left(P_{T}-P_{a}\right)}{(1-\alpha) \Sigma_{m} P_{T}} .
$$

The effective macroscopic total cross section $\bar{\Sigma}$ and the effective microscopic cross sections $\sigma_{p}$ and $\sigma_{m}$ are obtained 'on-the-fly' during the course of Monte Carlo random walks every time a neutron enters or collides the particle-dispersed medium. Other procedures are the same as the ordinary Monte Carlo calculations. Thus, the SLD method can be easily implemented into the production Monte Carlo calculation codes. The direct heterogeneous calculations, in which a large number of particles are explicitly distributed within a matrix material, require the time-consuming boundary search for determining the next particle into which a neutron enters. On the other hand, the SLD method can save the computation for the boundary search, thereby greatly enhancing the calculation efficiency for particle-dispersed media.

Although the slab thickness $L$ can be arbitrarily chosen, it is empirically known that $L=D$ mostly reproduces well the direct heterogeneous calculations for dispersed absorbing particles [2].

\section{Applications to neutron shielding calculation}

\subsection{Ordinary concrete containing $\mathrm{B}_{4} C$ particles}

The SLD method was applied to the neutron shielding calculation in an ordinary concrete containing $\mathrm{B}_{4} \mathrm{C}$ particles. A continuous energy Monte Carlo code MCNP 4C and a pointwise cross section library based on JENDL-3.3 [7] were used for the calculations. The density of the concrete was $2.30 \mathrm{~g} / \mathrm{cm}^{3}$. It is assumed that the isotopic composition of ${ }^{10} \mathrm{~B}$ is $100 \%$. The diameter of the particles depends on the fabrication specifications. This paper used a diameter of $0.0137 \mathrm{~mm}$ that was used for development of a radiation shielding concrete by Hazama Corporation [8]. The concentration of boron was $0.53 \mathrm{wt} . \%\left(12.1 \mathrm{~kg} / \mathrm{m}^{3}\right)$. The volume fraction of the particles was 0.00628 . The composition of the ordinary concrete is shown in Table 1 . The neutron beam leaking from a light-water reactor core was injected on the end surface of a 65 -cm-thick concrete. The angular distribution of the neutron beam was isotropic. Three types of calculations were performed; a direct heterogeneous calculation, a simple homogeneous calculation, and a homogeneous calculation with the SLD method. For the heterogeneous calculation, $96 \mathrm{~B}_{4} \mathrm{C}$ particles were randomly distributed in a cube with a side length of $0.274 \mathrm{~mm}$. Then, the cube was duplicated vertically and horizontally within the concrete slab. The neutron absorption rates of ${ }^{10} \mathrm{~B}$ and ${ }^{23} \mathrm{Na}$ were calculated. Table 2 shows the absorption rates per source neutron in the most upstream and most downstream 5-cm-thick layers for three calculations. Relative source particles per CPU time with three methods are compared in Table 2.

Table 1. Composition of the ordinary concrete.

\begin{tabular}{cccc}
\hline Element & $\begin{array}{c}\text { Composition } \\
\text { (wt.\%) }\end{array}$ & Element & $\begin{array}{c}\text { Composition } \\
\text { (wt.\%) }\end{array}$ \\
\hline $\mathrm{H}$ & 0.99 & $\mathrm{Si}$ & 33.4 \\
$\mathrm{C}$ & 0.26 & $\mathrm{~K}$ & 1.29 \\
$\mathrm{O}$ & 52.6 & $\mathrm{Ca}$ & 4.31 \\
$\mathrm{Na}$ & 1.59 & $\mathrm{Fe}$ & 1.38 \\
$\mathrm{Mg}$ & 0.22 & ${ }^{10} \mathrm{~B}$ & 0.53 \\
$\mathrm{Al}$ & 3.37 & & \\
\hline
\end{tabular}

Good agreement between the direct heterogeneous calculation and the SLD method was obtained both in the most upstream and most downstream layers. The calculation efficiency of the SLD method outperforms the direct heterogeneous calculation by a factor of 170 . The calculation efficiency of the heterogeneous calculation depends on the number of particles allocated in one cell. The more particles are allocated in a cell, the 
longer computation time is needed. The homogeneous calculation underestimates the absorption rate of ${ }^{23} \mathrm{Na}$ by $11 \%$ in the most upstream layer with respect to the heterogeneous system where the thermal neutron flux is relatively enhanced due to the self-shielding effect of the $\mathrm{B}_{4} \mathrm{C}$ particles. The homogeneous calculation neglects the self-shielding effect in the $\mathrm{B}_{4} \mathrm{C}$ particles, which underestimates the thermal neutron flux and in turn decreases the absorption of ${ }^{23} \mathrm{Na}$. The absorption rate of ${ }^{10} \mathrm{~B}$ by the homogeneous calculation agree well with the heterogeneous one, which is the consequence of the cancellation of the overestimation of the ${ }^{10} \mathrm{~B}$ 's effective absorption cross section in the particle-dispersed medium and the underestimation of the thermal neutron flux.

Table 2. Absorption rates of ${ }^{10} \mathrm{~B}$ and ${ }^{23} \mathrm{Na}$.

\begin{tabular}{|c|c|c|c|c|}
\hline & & hetero & SLD & homo \\
\hline \multirow{4}{*}{${ }^{10} \mathrm{~B}$} & upstream & $5.243 \mathrm{E}-1$ & $5.231 \mathrm{E}-1$ & $5.287 \mathrm{E}-1$ \\
\hline & & $(0.36 \%)^{*}$ & $(0.04 \%)$ & $(0.04 \%)$ \\
\hline & downstream & $1.086 \mathrm{E}-5$ & $1.079 \mathrm{E}-5$ & $1.079 \mathrm{E}-5$ \\
\hline & & $(1.95 \%)$ & $(0.23 \%)$ & $(0.22 \%)$ \\
\hline \multirow{4}{*}{${ }^{23} \mathrm{Na}$} & upstream & $1.302 \mathrm{E}-4$ & $1.305 \mathrm{E}-4$ & $1.168 \mathrm{E}-4$ \\
\hline & & $(0.36 \%)$ & $(0.04 \%)$ & $(0.04 \%)$ \\
\hline & downstream & $5.364 \mathrm{E}-9$ & $5.314 \mathrm{E}-9$ & $5.292 \mathrm{E}-9$ \\
\hline & & $(2.76 \%)$ & $(0.33 \%)$ & $(0.31 \%)$ \\
\hline \multicolumn{2}{|c|}{$\begin{array}{l}\text { source particles per } \\
\text { CPU time }\end{array}$} & 1 & 167 & 217 \\
\hline
\end{tabular}

*The values in the parentheses are fractional standard deviations.

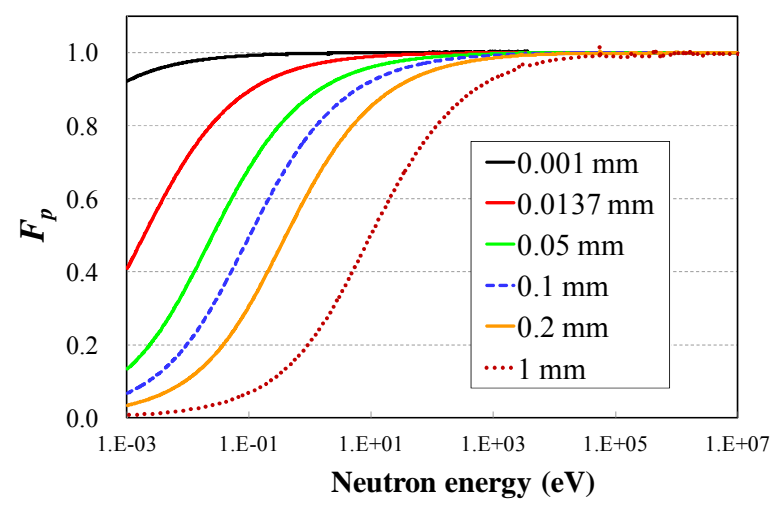

Figure 1. Heterogeneity effect on cross sections in the particle.

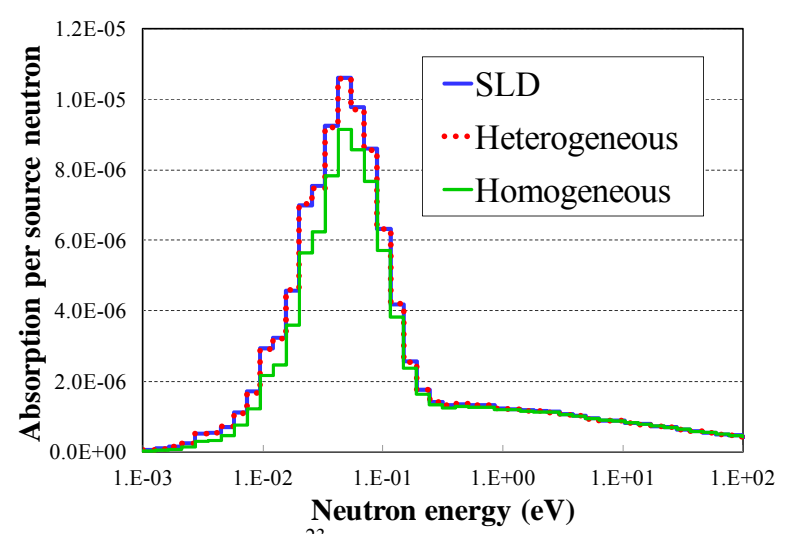

Figure 2. Absorption of ${ }^{23} \mathrm{Na}$ with SLD method, heterogeneous, and homogeneous calculations in the most upstream layer.
Figure 1 shows $F_{p}$ (Eq. (2)), the heterogeneity effect on cross sections in the $\mathrm{B}_{4} \mathrm{C}$ particles, for several particle diameters. The heterogeneity effect heavily depends on the diameter and neutron energy. For an energy range where $F_{p}$ is close to unity, the particle-dispersed medium can be treated as a homogeneous medium. For the diameter of $0.0137 \mathrm{~mm}$, the heterogeneity is not significant above $10 \mathrm{eV}$. Since most of the thermal neutrons are absorbed near the upstream surface of the concrete, the heterogeneity effect is not significant except near the upstream surface. As seen in Table 2, there is a minor difference between the absorption rates of ${ }^{23} \mathrm{Na}$ in the most downstream layer with the homogeneous and heterogeneous calculations.

In Figure 2, the absorption rates of ${ }^{23} \mathrm{Na}$ per source neutron in the most upstream layer with the SLD method, heterogeneous, and homogeneous calculations are compared for the particle diameter of $0.0137 \mathrm{~mm}$. The absorption rates with the SLD method and heterogeneous calculation are almost the same throughout the whole energy as far as one can see in Figure 2. The homogeneous calculation underestimates the absorption rate below $1 \mathrm{eV}$.

\subsection{Low-activation concrete containing $\mathrm{B}_{4} C$ particles}

Neutron shielding calculations were performed for a low-activation concrete containing $\mathrm{B}_{4} \mathrm{C}$ particles. The geometry and incident neutron spectrum were the same as in the previous section. The type '1-100 low-activation concrete' [6] with the density of 2.34 $\mathrm{g} / \mathrm{cm}^{3}$ was used in this paper. The major elements of the type 1-100 concrete are shown in Table 3.

Table 3. Composition of the low-activation concrete.

\begin{tabular}{cccc}
\hline Element & $\begin{array}{c}\text { Composition } \\
\text { (wt.\%) }\end{array}$ & Element & $\begin{array}{c}\text { Composition } \\
\text { (wt.\%) }\end{array}$ \\
\hline $\mathrm{H}$ & 0.84 & $\mathrm{Si}$ & 1.98 \\
$\mathrm{C}$ & 9.29 & $\mathrm{Ca}$ & 35.5 \\
$\mathrm{O}$ & 49.3 & $\mathrm{Fe}$ & 0.40 \\
$\mathrm{Na}$ & 0.04 & ${ }^{10} \mathrm{~B}$ & 0.0724 \\
$\mathrm{Mg}$ & 0.31 & $\mathrm{Co}$ & 0.00615 \\
$\mathrm{Al}$ & 0.30 & $\mathrm{Eu}$ & 0.00124 \\
\hline
\end{tabular}

Table 4. Absorption rates of ${ }^{10} \mathrm{~B},{ }^{59} \mathrm{Co}$, and ${ }^{151} \mathrm{Eu}$.

\begin{tabular}{|c|c|c|c|c|}
\hline & & hetero & SLD & homo \\
\hline \multirow{4}{*}{${ }^{10} \mathrm{~B}$} & upstream & $3.460 \mathrm{E}-1$ & $3.459 \mathrm{E}-1$ & $3.620 \mathrm{E}-1$ \\
\hline & \multirow{3}{*}{ downstream } & $(0.39 \%)^{*}$ & $(0.06 \%)$ & $(0.06 \%)$ \\
\hline & & $1.149 \mathrm{E}-5$ & $1.141 \mathrm{E}-5$ & $1.144 \mathrm{E}-5$ \\
\hline & & $(1.52 \%)$ & $(0.26 \%)$ & $(0.25 \%)$ \\
\hline \multirow{4}{*}{${ }^{59} \mathrm{Co}$} & \multirow{4}{*}{$\begin{array}{l}\text { upstream } \\
\text { downstream }\end{array}$} & $7.922 \mathrm{E}-5$ & $7.929 \mathrm{E}-5$ & $7.237 \mathrm{E}-5$ \\
\hline & & $(0.71 \%)$ & $(0.12 \%)$ & $(0.12 \%)$ \\
\hline & & $5.021 \mathrm{E}-9$ & $5.053 \mathrm{E}-9$ & 4.888E-9 \\
\hline & & $(1.60 \%)$ & $(0.27 \%)$ & $(0.26 \%)$ \\
\hline \multirow{4}{*}{${ }^{151} \mathrm{Eu}$} & upstream & $4.808 \mathrm{E}-4$ & $4.798 \mathrm{E}-4$ & $4.202 \mathrm{E}-4$ \\
\hline & \multirow{3}{*}{ downstream } & $(0.45 \%)$ & $(0.08 \%)$ & $(0.07 \%)$ \\
\hline & & $1.788 \mathrm{E}-8$ & $1.775 \mathrm{E}-8$ & $1.659 \mathrm{E}-8$ \\
\hline & & $(1.51 \%)$ & $(0.26 \%)$ & $(0.25 \%)$ \\
\hline \multicolumn{2}{|c|}{$\begin{array}{l}\text { source particles per } \\
\text { CPU time }\end{array}$} & 1 & 90 & 109 \\
\hline
\end{tabular}


Again, the diameter of the $\mathrm{B}_{4} \mathrm{C}$ particles was 0.0137 $\mathrm{mm}$. The volume fraction of the particles was 0.000882 . For the heterogeneous calculation, $96 \mathrm{~B}_{4} \mathrm{C}$ particles were randomly distributed in a cube with a side length of $0.527 \mathrm{~mm}$. Table 4 shows the absorption rates in the most upstream and most downstream 5-cm-thick layer for the heterogeneous, homogeneous and SLD method calculations. There are good agreements between the heterogeneous and SLD method calculations. The SLD method is about 90 times faster than the direct heterogeneous calculation. The homogeneous calculation underestimates the absorption rates of ${ }^{59} \mathrm{Co}$ and ${ }^{151} \mathrm{Eu}$ in the most upstream layer by $9 \%$ and $14 \%$, respectively. In Figure 3, the absorption rates of ${ }^{151} \mathrm{Eu}$ per source neutron in the most upstream layer with the SLD method, heterogeneous, and homogeneous calculations are compared. A significant difference of the ${ }^{151} \mathrm{Eu}$ absorption between the heterogeneous and homogeneous calculations is found below $0.1 \mathrm{eV}$.

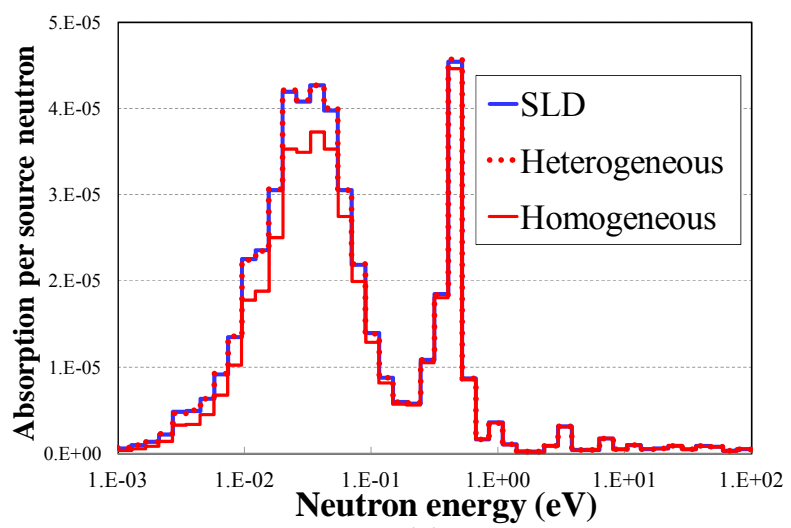

Figure 3. Absorption of ${ }^{151} \mathrm{Eu}$ with SLD method, heterogeneous, and homogeneous calculations in the most upstream layer.

\section{Conclusions}

This paper presents the first application of the SLD method to neutron shielding calculations. It is confirmed that the SLD method can precisely reproduce direct heterogeneous calculations for deep penetration calculations of concrete shields containing $\mathrm{B}_{4} \mathrm{C}$ particles. A direct heterogeneous calculation with Monte Carlo method where a large number of particles are explicitly distributed in a matrix material requires a large amount of computation burden especially for small size particles. If the diameter of the particles is small enough, the particle-dispersed medium can be treated as a homogeneous medium without reducing accuracy of the calculation. However, even for a concrete shield containing particles with the diameter of $10 \mu \mathrm{m}$, the heterogeneity effect is significant in terms of the activations of $\mathrm{Na}, \mathrm{Co}$, and $\mathrm{Eu}$ etc. The activations of these elements are important for assuring radiation control or clearance level of decommissioning. The SLD method calculates effective homogenized cross sections for particle-dispersed media 'on-the-fly' during the course of Monte Carlo random walks. Since the particle-dispersed media can be treated as homogeneous media in the SLD method, the time-consuming boundary search in the particle-dispersed media can be avoided. The SLD method outperforms the direct heterogeneous calculations by a factor of 100 in the numerical tests of this paper. The SLD method is expected to be applicable to a variety of shielding calculations where small particles are dispersed.

\section{References}

[1] V.M. Shmakov, V.D. Lyutov and V.F. Dean, Effective cross section for calculations of criticality of dispersed media, Proc. the 2000 American Nuclear Society International Topical Meeting on Advances in Reactor Physics and Mathematics and Computation into the Next Millennium (PHYSOR2000), Pittsburgh, PA, USA, May 7-11, 2000, (2000).

[2] T. Yamamoto, Y. Miyoshi and T. Takeda, Extension of effective cross section calculation method for neutron transport calculations in particle-dispersed media, J. Nucl. Sci. Technol. 43 (2006), pp. 77-87.

[3] T. Yamamoto, Extension of cross section homogenization method for particle-dispersed media to layered particles, Ann. Nucl. Energy. 33 (2006), pp. 804-812.

[4] T. Yamamoto, Study on double heterogeneity effect of Pu-rich agglomerates in mixed oxide fuel using cross section homogenization method for particle-dispersed media, Ann. Nucl. Energy. 37 (2010), pp. 398-405.

[5] J.F. Briesmeister, Ed., MCNP-A general Monte Carlo N-particle Transport Code Version 4C, LA-13709-M (2000).

[6] M. Kinno, K. Kimura, Y. Fujikura, H. Nishida, N. Katayose, T. Mori, R. Yoshino, T. Tanosaki, K. Ichitsubo, M. Nakata, T. Ogata, M. Uematsu, K. Hayashi, M. Sato, M. Sakakibara, S. Ito, K. Yamaguchi, M. Satou and A. Hasegawa, Low-activation multilayer shielding structure of light water reactor using various types of low-activation concrete, Prog. Nucl. Sci. Technol. 1 (2011), pp. 28-31.

[7] K. Shibata, T. Kawano, T. Nakagawa et al., Japanese evaluated nuclear data library version 3 revision-3: JENDL-3.3, J. Nucl. Sci. Technol. 39 (2002), pp. 1125-1136.

[8] K. Okuno, H. Yamada and A. Yagenji, Studies on boron containing concrete for radiation shielding, Abstracts of Architectural Institute of Japan, (2000). [in Japanese] 